\title{
Microbiome and production performance
}

\section{POULTRY GASTROINTESTINAL MICROBIOME CHANGES DURING ONTOGENESIS}

\section{V.I. FISININ'1, G.Yu. LAPTEV ${ }^{2}$, I.N. NIKONOV ${ }^{2}$, L.A. IL'INA ${ }^{2}$, E.A. YILDIRIM ${ }^{2}$, V.A. FILIPPOVA ${ }^{2}$, N.I. NOVIKOVA ${ }^{2}$, A.A. GROZINA ${ }^{1}$, T.A. EGOROVA ${ }^{1}$, T.N. LENKOVA1, V.A. MANUKYAN', I.A. EGOROV1}

\author{
${ }^{1}$ All-Russian Research and Technological Poultry Institute, Federal Agency of Scientific Organizations, 10, ul. Pti- \\ tsegradskaya, Sergiev Posad, Moscow Province, 141311 Russia, e-mail olga@vnitip.ru; \\ $2 J S C$ «Biotrof», Kolpino, St. Petersburg, 192288 Russia, e-mail nikonov@biotrof.ru \\ Acknowledgements: \\ Supported by grant from Russian Science Foundation (project № 14-16-00140 «Modern views on the intestinal \\ microflora of poultry in different diets: molecular genetic approaches») \\ Received September 13, 2016
}

Abstract

Microorganisms which inhabit gut play great role in providing with nutrients, antibiotics, hormones and vitamins necessary for poultry health and performance. Therefore study of gut microbiome changes during ontogenesis seems to be essential. The structure of gut microflora in poultry embryos is of particular interest and debated because of very few publications on the problem. Despite embryo intestine is commonly considered sterile there are several reports on gut colonization by microorganisms in embryos during ontogenesis. Using T-RFLP (Terminal Restriction Fragment Length Polymorphism) analysis to generate a fingerprint of a microbial community we compared gut flora in chick embryos on days 6 and 17 to those in 26-day, 150-day and 300-day old Hisex White layers. Unlike accepted view, a high biodiversity was seen in embryo gut with Enterobacteriaceae (Escherichia coli mainly) predominated. Clostridia, Bacteroides, Negativicutes, Actinomycetales, Bifidocteriales were also found in contrast to earlier reports of their presence only in chicks at hatching and in adult poultry gut. Moreover, in the embryo gut we found the causal agents of dangerous animal disease, Burkholderia sp., Pseudomonas sp., Salmonella sp., Klebsiella sp. and Rickettsiales bacteria. Interestingly, the embryo gut biodiversity on day 6 was higher as compared to day 17 (75 \pm 2.75 phylotypes vs $30 \pm 1.20$ phylotypes). In the layers aged 26,150 and 300 days the diversity was much higher (over $175 \pm 8.12$ phylotypes) as compared to embryos due to new members involved into gut bacterial community. Moreover, the poultry aged 300 days was lower both in the total diversity and in the percentage of unidentified microorganisms when compared to 26-day and 150-day old hens. In the adults, the predominating microbial taxa changed, in particular, Clostridia and Negativicutes became more abundant whereas Bacillales and Bifidobacteriales were depressed. Our findings indicate gut colonization by Lactobacilales and pathogenic Listeria sp., Pantoea sp., Enterobacter sp., Mycoplasma sp., Acinetobacter sp., Pasteurellaceae, Campylobacteraceae, Fusobacteria which occurred during ontogenesis. Thus the gut microbiome formation starts in embryo which is important for hatching and growing healthy poultry.

Keywords: gut microflora, caecum, ontogenesis, hens, embryo, T-RFLP

Microorganisms which inhabit the gastrointestinal tract (GIT), by using their own cellulosolytic and amylolytic enzymes, completely absent in poultry, provide the host with nutrients, vitamins, antibiotics, proteins, hormones and other compounds involved in metabolism [1-4]. According to traditional concepts, microbiocenosis of embryonal digestive system is sterile, and its colonization by microorganisms takes place after chicken hatching [5-7]. Bacteria inhabiting poultry intestine normally include bifidobacteria, streptococci, lactobacilli, lactate fermenting bacteria, eubacteria, bacteroides and enterobacteria $[1,8,9]$.

Bacterial community of digestive tract undergoes subsequent changes throughout life of a bird, related to a number of factors, the main of which are growth and development of digestive tract, feeding regime and feed composition. 
At that, intestinal microorganisms act as a highly sensitive indicator system. It should be noted that the change of ecological balance between obligatory microorganisms of digestive tract do not always have a positive impact on metabolic processes and health of a bird [10]. In this context, studies of qualitative and quantitative composition of GIT microbiota in ontogenesis are worthwhile.

Up to the 1990s, studies of microorganisms in various ecosystems were limited to examination of strains cultivated on artificial media. Development of metagenomic methods, without a necessity to cultivate microorganisms, allowed us to broaden our understanding of composition of microbiota [11, 12]. This is of crucial significance, as up to $99 \%$ of biosphere microorganisms are unculturable on artificial media, but may play an important ecological role. We failed to find any data on composition of chicken embryo GIT microbiome in available literature.

For the first time, we have analyzed the composition of chicken GIT bacterial community in ontogenesis, from an embryo to an adult bird, using T-RFLP (terminal restriction fragment length polymorphism) method. A wide taxonomic composition of embryonal intestine bacteria has been demonstrated, including opportunistic strains, pathogens and unculturable microorganisms.

The work focuses on examination of succession in a bacterial community of poultry GIT in ontogenesis, using T-RFLP method.

Technique. Three samples of GIT content of 6 and 12-day old chicken embryos and three samples from cecum of Hisex White adult 26, 150 and 300day old laying hens were collected (hatching house of the All-Russian Research and Technological Poultry Institute - VNITIP, Zagorskoe EPH VNITIP vivarium, Moscow region). The poultry were fed manually, ad libitum with dry complete feed according to the cross standards. The birds were kept in cage batteries (Big Dutchman, Germany) in groups of 35 individuals, with no gender separation, in compliance with all technological parameters according to the norms of VNITIP. Sampling and sample preparation was performed in strict compliance with sterility in accordance with the established requirements [13].

Total DNA was isolated from the samples using Genomic DNA Purification Kit (Fermentas, Inc., Lithuania) according to manufacturer's recommendations. T-RFLP analysis was used for examination of bacterial community composition. PCR was performed using a Verity DNA amplificator (Life Technologies, Inc., USA) and 63F primer (CAGGCCTAACACATGCAAGTC) with the 5' end marked (fluorophore WellRed D4, Beckman Coulter, USA), and 1492R primer (TACGGHTA-CCTTGTTACGACTT). Fluorescence-labelled amplicons of 16S rRNA gene were purified by a standard methodology [14]. The obtained amplicons (30-50 ng) were treated with endonucleases HaeIII, HhaI and MspI, according to the manufacturer's recommendation (Fermentas, Lithuania). Restriction products were analyzed on a CEQ 8000 sequencer (Beckman Coulter, USA). Phylogenetic status of bacteria was determined using Fragment Sorter software (http://www.oardc.ohiostate.edu/trflpfragsort/index.php).

Statistical processing of the results was performed by means of analysis of variance using Microsoft Excel 2010.

Results. It is generally believed that the GIT of avian embryos is sterile $[1,15,16]$, and the formation of digestive system microbiocenosis takes place after hatching as due to a contact with the environment [5-7]. However, there is evidence obtained by classical microbiology methods $[17,18]$ and real time PCR [19] which indicates the ability of microorganisms to colonize GIT of birds still inside an egg, at the embryonic development stage.

Using T-RFLP analysis, we have established that embryonal GIT bacterial community was characterized by significant taxonomic diversity (Table). Bi- 
odiversity of microorganisms in microbiocoenosis of chicken embryo GIT content on day 6 of incubation was much more significant than that on day 16 . Members of family Enterobacteriaceae, typical of poultry intestine microflora, were predominant, mainly Escherichia coli. Interestingly, their number was twice

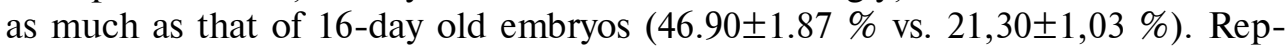
resentatives of class Clostridia (families Lachnospiraceae, Eubacteriaceae, etc.), phylum Bacteroidetes, orders Negativicutes, Actinomycetales, Bifidobacteriales were also identifies among the embryonal GIT indigenous microflora, which were previously detected in hatched and adult chickens [20]. Amylo- and proteolytic bacteria of order Actinomycetales, cellulolytic microorganisms of class Clostridia, phylum Bacteroidetes, inhabiting GIT of hatched chickens and adult birds, play an important role in metabolism due to active participation in fermentation of protein, starch and polysaccharides of feeds.

Microorganisms of order Bifidobacteriales, producing organic acids and bacteriocins in GIT of birds, can ensure colonization resistance of a microbiotopes to pathogens, and produce essential nutrients [1]. We heve observed 130fold increase in bifidobacteria amount in embryonal intestine during incubation

Interestingly, metagenomic community of GIT of 16-day old embryos has shown absence of autochthonous symbiotic intestine microflora, typical in birds, i.e. facultative anaerobic bacteria of order Lactobacillales, while the proportion of these bacteria in GIT of 6-day old chicken embryos was high $(10.11 \pm 0.42 \%)$. Most bacteria of order Lactobacillales are capable of active acid production, so they ensure competitive exclusion of pathogens in GIT of hatched chickens and adult birds [1]. With regard to order Lactobacillales, the proportion of microorganisms of genus Lactobacillus was $9.30 \pm 0.39 \%$, of genus Pediococcus $-0.66 \pm 0.02 \%$ and Trichococcus $-0.150 \pm 0.006 \%$. A high percentage of order Bacillales representatives, mostly bacteria of genus Bacillus, were detected in GIT of embryos. The majority of them are capable of poultry digestive tract colonization, synthesis of organic acids, bacteriocins, antibiotics and enzymes, active participation in metabolic processes of various nutritious substrates $[18,19]$. Apart from that, several unidentified bacterial genotypes were observed.

Pathogenic and opportunistic bacteria, causative agents of dangerous diseases in animals of genera Burkholderia, Pseudomonas, Salmonella, Klebsiella, order Rickettsiales, etc. (Table) were detected in metagenomic community of 6day old embryo intestine; on day 16 of incubation a colonization of GIT with new microorganisms of genera Staphylococcus, Morganella, Bordetella was observed, which have possibly entered through eggshell pores.

It should be noted that E. coli and bacteria of genera Staphylococcus and Pseudomonas, detected by us in GIT of embryos during incubation, may cause omphalitis (i.e. inflammation of the navel and yolk sac), a dangerous disease which is the main reason of death in chickens from hatching to day 14 of life [21]. Microorganisms of genus Bordetella cause respiratory tract diseases in poultry, mostly in young chickens [22]. Bacteria of order Rickettsiales are pathogens, transmitted by representatives of Arthropoda. Presumably, development of the said diseases is caused by unfavorable composition of GIT microflora, i.e. increase in the number of the pathogens during embryonic development.

The results obtained by us using T-RFLP analysis correspond to the data of Z. Babaca [18]. Using classical microbiological methods, the author has examined more than 3000 samples of chicken embryo GIT content from incubators of three poultry farms, in order to identify the reasons of the mass death of embryos, and has detected pathogenic bacteria E. coli (18.28 \%), Staphylococcus $(14.10 \%)$, Pseudomonas (11.75\%) and Klebsiella sp. in the samples. It has been also reported that microflora of laying hens plays a key role in formation of em- 
bryo GIT pathogenic microflora [17)]. Using the real time PCR, it has been shown that content of these microorganisms in GIT of laying hens, artificially contaminated with Campylobacter coli, amounts to $4.35-5.65$ thous. cells/g of body weight [17]. Our results are indicative of GIT colonization by microorganisms as early as at the embryonic development stage.

The proportion and number of bacterial taxa in embryo GIT and cecum of Hisex White laying hens of various age $(X \pm x$, FSUE Zagorskoe EPH VNITIP vivarium, Moscow region)

\begin{tabular}{|c|c|c|c|c|c|}
\hline \multirow{3}{*}{$\begin{array}{l}\text { Taxonomic } \\
\text { group of bacteria }\end{array}$} & \multicolumn{5}{|c|}{ Frequency $(\%)$ and total number of taxa in a group } \\
\hline & \multicolumn{2}{|c|}{ embryo, age } & \multicolumn{3}{|c|}{ bird, age } \\
\hline & day 6 & day 17 & day 26 & day 150 & day 300 \\
\hline Phylum Firmicutes & $25.01 \pm 1.03$ & $10.01 \pm 0.40$ & $55.13 \pm 2.58$ & $56.39 \pm 2.79$ & $61.02 \pm 2.88$ \\
\hline Class Clostridia & $5.5 \pm 0.17$ & $1.10 \pm 0.03$ & $31.82 \pm 1.27$ & $20.37 \pm 0.98$ & $17.35 \pm 0.84$ \\
\hline Family Lachnospiraceae & $0.08 \pm 0.01$ & $1.10 \pm 0.03$ & $4.61 \pm 0.18$ & $1.96 \pm 0.08$ & $1.85 \pm 0.07$ \\
\hline Family Eubacteriaceae & $0.32 \pm 0.01$ & N/D & $5.43 \pm 0.20$ & $5.58 \pm 0.23$ & $3.61 \pm 0.12$ \\
\hline Family Ruminococcaceae & $0.38 \pm 0.01$ & $\mathrm{~N} / \mathrm{D}$ & $8.72 \pm 0.35$ & $5.18 \pm 0.20$ & $5.95 \pm 0.22$ \\
\hline Family Clostridiaceae & $1.70 \pm 0.06$ & $\mathrm{~N} / \mathrm{D}$ & $11.40 \pm 0.45$ & $6.19 \pm 0.28$ & $5.20 \pm 0.21$ \\
\hline Family Peptococcaceae & N/D & $\mathrm{N} / \mathrm{D}$ & $1.66 \pm 0.04$ & $1.46 \pm 0.06$ & $0.74 \pm 0.03$ \\
\hline Family Syntrophomonadaceae & $2.30 \pm 0.04$ & $\mathrm{~N} / \mathrm{D}$ & N/D & N/D & N/D \\
\hline Class Negativicutes & $0.70 \pm 0.03$ & N/D & $12.70 \pm 0.58$ & $7.91 \pm 0.39$ & $8.77 \pm 0.39$ \\
\hline Class Bacilli & $18.81 \pm 0.84$ & $8.91 \pm 0.36$ & $10.61 \pm 0.76$ & $28.11 \pm 1.35$ & $34.90 \pm 1.25$ \\
\hline Order Lactobacillales & $10.11 \pm 0.42$ & N/D & $7.28 \pm 0.33$ & $22.56 \pm 0.95$ & $32.48 \pm 1.22$ \\
\hline Lactobacillus sp. & $9.30 \pm 0.39$ & $\mathrm{~N} / \mathrm{D}$ & $5.14 \pm 0.15$ & $16.00 \pm 0.75$ & $26.10 \pm 1.10$ \\
\hline Enterococcus sp. & N/D & $\mathrm{N} / \mathrm{D}$ & $0.61 \pm 0.03$ & $4.18 \pm 0.18$ & $3.06 \pm 0.14$ \\
\hline Leuconostoc sp. & N/D & $\mathrm{N} / \mathrm{D}$ & $1.11 \pm 0.04$ & $1.11 \pm 0.03$ & $0.49 \pm 0.02$ \\
\hline Pediococcus sp. & $0.66 \pm 0.02$ & $\mathrm{~N} / \mathrm{D}$ & $0.22 \pm 0.01$ & $0.67 \pm 0.02$ & $1.75 \pm 0.05$ \\
\hline Weissella sp. & N/D & $\mathrm{N} / \mathrm{D}$ & $0.20 \pm 0.01$ & $0.60 \pm 0.03$ & $1.08 \pm 0.04$ \\
\hline Trichococcus sp. & $0.15 \pm 0.01$ & N/D & N/D & N/D & N/D \\
\hline Order Bacillales & $8.70 \pm 0.41$ & $8.91 \pm 0.36$ & $3.35 \pm 0.15$ & $5.08 \pm 0.19$ & $2.33 \pm 0.11$ \\
\hline Alicyclobacillus sp. & $0.16 \pm 0.01$ & $0.50 \pm 0.02$ & $0.10 \pm 0.01$ & N/D & $0.08 \pm 0.00$ \\
\hline Brevibacillus sp. & $0.34 \pm 0.01$ & N/D & N/D & N/D & N/D \\
\hline Bacillus sp. & $7.10 \pm 0.33$ & $6.79 \pm 0.28$ & $2.05 \pm 0.09$ & $2.46 \pm 0.11$ & $1.37 \pm 0.05$ \\
\hline Paenibacillus sp. & $1.10 \pm 0.04$ & $1.21 \pm 0.03$ & $0.55 \pm 0.02$ & $0.99 \pm 0.02$ & N/D \\
\hline Staphylococcus sp. & N/D & $0.41 \pm 0.01$ & $0.65 \pm 0.03$ & $1.46 \pm 0.05$ & $0.88 \pm 0.01$ \\
\hline Listeria $\mathrm{sp}$ & N/D & N/D & N/D & $0.17 \pm 0.01$ & N/D \\
\hline Phylum Actinobacteria & $4.53 \pm 0.21$ & $34.2 \pm 1.02$ & $4.12 \pm 0.17$ & $3.18 \pm 0.12$ & $2.21 \pm 0.11$ \\
\hline Order Actinomycetales & $4.38 \pm 0.19$ & $13.58 \pm 0.65$ & $3.74 \pm 0.14$ & $2.63 \pm 0.12$ & $2.05 \pm 0.09$ \\
\hline Order Bifidobacteriales & $0.15 \pm 0.01$ & $20.62 \pm 0.89$ & $0.38 \pm 0.02$ & $0.55 \pm 0.02$ & $0.16 \pm 0.01$ \\
\hline Phylum Proteobacteria & $34.83 \pm 1.62$ & $49.60 \pm 2.32$ & $9.88 \pm 0.44$ & $9.24 \pm 0.36$ & $9.44 \pm 0.41$ \\
\hline Family Enterobacteriaceae & $22.35 \pm 0.98$ & $47.26 \pm 1.98$ & $1.07 \pm 0.04$ & $3.94 \pm 0.14$ & $2.63 \pm 0.10$ \\
\hline Pantoea sp. & N/D & N/D & $0.17 \pm 0.01$ & $0.76 \pm 0.03$ & $0.73 \pm 0.03$ \\
\hline Salmonella sp. & $0.26 \pm 0.01$ & N/D & $0.24 \pm 0.01$ & $0.21 \pm 0.01$ & $0.34 \pm 0.01$ \\
\hline Morganella sp. & N/D & $0.36 \pm 0.02$ & N/D & N/D & N/D \\
\hline Enterobacter sp. & $\mathrm{N} / \mathrm{D}$ & $\mathrm{N} / \mathrm{D}$ & $0.11 \pm 0.01$ & $1.39 \pm 0.05$ & $1.49 \pm 0.06$ \\
\hline $\begin{array}{l}\text { Escherichia coli } \\
\text { other (Citrobacter } \mathrm{s}\end{array}$ & $21.30 \pm 1.03$ & $46.90 \pm 1.87$ & $0.29 \pm 0.01$ & $0.98 \pm 0.04$ & $0.06 \pm 0.01$ \\
\hline $\begin{array}{l}\text { sp., Rahnella sp., Serratia sp., } \\
\text { Yersinia sp.) }\end{array}$ & $0.79 \pm 0.01$ & $\mathrm{~N} / \mathrm{D}$ & $0.26 \pm 0.01$ & $0.60 \pm 0.02$ & $0.01 \pm 0.00$ \\
\hline Order Burkholderiales & $0.31 \pm 0.01$ & $2.00 \pm 0.08$ & $\mathrm{~N} / \mathrm{D}$ & N/D & $\mathrm{N} / \mathrm{D}$ \\
\hline Burkholderia sp. & $0.31 \pm 0.01$ & $0.94 \pm 0.04$ & $\mathrm{~N} / \mathrm{D}$ & N/D & $\mathrm{N} / \mathrm{D}$ \\
\hline Bordetella sp. & N/D & $1.09 \pm 0.03$ & N/D & N/D & N/D \\
\hline Order Pseudomonadales & $2.62 \pm 0.10$ & $\mathrm{~N} / \mathrm{D}$ & $7.42 \pm 0.29$ & $3.69 \pm 0.14$ & $2.7 \pm 0.12$ \\
\hline Acinetobacter sp. & N/D & $\mathrm{N} / \mathrm{D}$ & $2.90 \pm 0.13$ & $0.50 \pm 0.02$ & $0.55 \pm 0.02$ \\
\hline Pseudomonas sp. & $2.62 \pm 0.10$ & $\mathrm{~N} / \mathrm{D}$ & $4.52 \pm 0.20$ & $3.19 \pm 0.14$ & $2.15 \pm 0.12$ \\
\hline Family Caulobacteraceae & & & & & \\
\hline (Brevundimonas sp.) & $9.44 \pm 0.42$ & $0.31 \pm 0.02$ & $\mathrm{~N} / \mathrm{D}$ & $\mathrm{N} / \mathrm{D}$ & $\mathrm{N} / \mathrm{D}$ \\
\hline $\begin{array}{l}\text { Family Pasteurellaceae } \\
\text { (Pasteurella sp.. Haemophilus sp.) }\end{array}$ & & & & & \\
\hline $\begin{array}{l}\text { (Pasteurella sp., Haemophilus sp.) } \\
\text { Family Campylobacteraceae }\end{array}$ & $\mathrm{N} / \mathrm{D}$ & $\mathrm{N} / \mathrm{D}$ & $0.48 \pm 0.03$ & $0.92 \pm 0.02$ & $0.69 \pm 0.02$ \\
\hline (Campylobacter sp., Arcobacter sp.) & N/D & $\mathrm{N} / \mathrm{D}$ & $0.91 \pm 0.04$ & $0.69 \pm 0.03$ & $3.42 \pm 0.60$ \\
\hline Order Rickettsiales & $0.11 \pm 0.01$ & $\mathrm{~N} / \mathrm{D}$ & N/D & N/D & N/D \\
\hline Phylum Tenericutes (Mycoplasma sp.) & N/D & $\mathrm{N} / \mathrm{D}$ & $1.16 \pm 0.05$ & $1.01 \pm 0.03$ & $1.13 \pm 0.04$ \\
\hline Phylum Bacteroidetes & $5.42 \pm 0.22$ & $\mathrm{~N} / \mathrm{D}$ & $8.88 \pm 0.35$ & $9.68 \pm 0.44$ & $9.24 \pm 0.39$ \\
\hline Phylum Fusobacteria & N/D & N/D & $1.39 \pm 0.04$ & $2.21 \pm 0.12$ & $2.71 \pm 0.33$ \\
\hline Unidentified bacteria & $30.21 \pm 1.42$ & $6.19 \pm 0.28$ & $19.44 \pm 0.56$ & $18.29 \pm 0.86$ & $14.25 \pm 0.69$ \\
\hline Total number of phylotypes, pcs & $75 \pm 2.75$ & $30 \pm 1.20$ & $224 \pm 10.23$ & $255 \pm 12.46$ & $175 \pm 8.12$ \\
\hline
\end{tabular}

We found the taxonomic composition of poultry cecum content to be much more diverse and significantly different from that of embryo GIT. First of 
all, regardless of the age of chickens, representatives of phylum Firmicutes (above $55.13 \%$ ), the proportion of which increased with age, rather than microorganisms of family Enterobacteriaceae, were taxonomic dominants. In 26-day old chickens and 150 and 300-day old laying hens the representatives of class Clostridia predominated, which mostly includes bacteria of families Eubacteriaceae, Ruminococcaceae, Peptococcaceae, Clostridiaceae, Lachnospiraceae with celluloseand amylolytic properties, content of which decreased with age. The number of bacteria with similar properties from phylum Bacteroidetes was not related to the age of laying hens and was higher than that in embryo GIT. Birds have little, if any, digestive enzymes of their own, required for cellulose and other non-starch polysaccharides cleavage, so the role of the said microorganisms in digestion of chickens is extremely important [1,2].

As compared to embryos, the number of bacteria from class Negativicutes, which play an important role in digestion by fermentation of organic acids, including lactate, with formation of volatile fatty acids necessary for energy supply [2], increased significantly in adult chickens.

The proportion of microorganisms of orders Bacillales and Bifidobacteriales, characterized by high antagonistic activity towards pathogenic microflora, decreased with age and was significantly lower than that of embryo GIT. An inverse trend was observed for lactobacilli of order Lactobacillales, the largest proportion of which was detected in 300-day old chickens. Diversity of lactobacilli in adult chickens was higher than in enbryos, and included the representatives of genera Weissella, Leuconostoc and Enterococcus as well.

The diversity of pathogens in 26-day chickens and 150 and 300-day old adults was significantly higher than that in embryos. Poultry GIT was colonized by new pathogens of genera Listeria, Pantoea, Enterobacter, Mycoplasma, Acinetobacter, families Pasteurellaceae, Campylobacteraceae, phylum Fusobacteria. Some microorganisms of the listed taxa (Pantoea, Enterobacter, Acinetobacter) cause intestine dysbiotic disorders in mammals and birds, while the other (genus Mycoplasma, family Pasteurellaceae) are mostly detected in the respiratory tract in birds and are considered as causative agents of respiratory diseases. The fact that bacteria of phylum Fusobacteria, which were previously considered as typical inhabitants of the rumen of ruminants, are found in poultry cecum is of significant interest [23]. It should be noted that the presence of several listed pathogens in poultry GIT was previously reported only based on molecular genetic studies [24, 25].

Interestingly, some pathogens detected in embryo GIT, including bacteria of orders Burkholderiales and Rickettsiales and genus Brevundimonas, have not been observed in 26-day old chickens, as well as in 150 and 300-day old laying hens. At that, the number of unidentified bacteries decreased with age of a bird.

Thus, using T-RFLP analysis, highly abundant taxonomic diversity of bacteria has been detected in the gastrointestinal tract (GIT) of chicken embryos, including the representatives of indigenous GIT normal flora of hatched and adult chickens, opportunistic and pathogenic microflora, and unculturable microorganisms. Taxonomic diversity in chicken GIT content on day 6 of incubation was much more significant than that on day 16 . The bacterial community of poultry GIT develops during ontogenesis, and new microorganisms appear. Cecum community in 26-day old chicken, as well as 150 and 300-day old poultry is represented by a wide variety of microorganisms, including opportunistic and pathogenic ones. In conclusion, development of microbioecological system, as GIT content with inhabiting microflora, starts in poultry as early as at the embryonic development stage. Presumably, the structure of embryo GIT microbiotope forms under the influence of a laying hen microflora by vertical transfer due to bacterial trans- 
location. Presumably, microflora that colonizes chicken embryo GIT enters through eggshell pores. At that, microorganisms in embryo GIT act as a basis of the forming initial intestine biocenosis in hatched chickens, which in many ways determines their viability, resistance to pathogens and development.

\section{R E F EREN CES}

1. Ti m o s h k o M.A. Mikroflora pishchevaritel'nogo trakta sel'skokhozyaistvennykh zhivotnykh [Microflora of alimentary canal in farm animals]. Kishinev, 1990 (in Russ.).

2. Tarakanov B.V. Metody issledovaniya mikroflory pishchevaritel'nogo trakta sel'skokhozyaistvennykh zhivotnykh $i$ ptitsy [Study of microflora in alimentary canal of farm animals methods]. Moscow, 2006 (in Russ.).

3. Salanitro J., Fairchilds I., Zgornicki Y. Isolation, culture characteristics, and identification of anaerobic bacteria from the chicken cecum. Appl. Microbiol., 1974, 27: 678-687.

4. Stanley D., Hughes R.J., M o ore R.J. Microbiota of the chicken gastrointestinal tract: influence on health, productivity and disease. Appl. Microbiol. Biotechnol., 2014, 98: 4301-4309 (doi: 10.1007/s00253-014-5646-2).

5. M e a d G.C. Microbes of the avian cecum: types present and substrates utilized. J. Exp. Zool. Suppl., 1989, 3: 48-54.

6. A mit R o m a ch E., S k la n D., U n i Z. Microflora ecology of the chicken intestine using 16S ribosomal DNA primers. Poultry Sci., 2004, 83: 1093-1098.

7. Dibner J.J., Richards J.D., Knight C.D. Microbial imprinting in gut development and Health. J. Appl. Poultry Res., 2008, 17: 174-188 (doi: 10.3382/japr.2007-00100).

8. B a rne s E. The intestinal microbiota of poultry and game birds during life and after storage. J. Appl. Bacteriol., 1979, 46: 407-419.

9. Me a d G.C. Microbes of the avian cecum: types present and substrates utilized. J. Exp. Zool., 1989, 3: 48-54 (doi: 10.1002/jez.1402520508).

10. To rok V., O phe l-Kelle r K., L o o M., Hughes R. Application of methods for identifying broiler chicken gut bacterial species linked with increased energy metabolism. Appl. Environ. Microbiol., 2008, 74(3): 783-791 (doi: 10.1128/AEM.01384-07).

11. Park S.H., Le e S.I., R i cke S.C. Microbial populations in naked neck chicken ceca raised on pasture flock fed with commercial yeast cell wall prebiotics via an Illumina MiSeq Platform. PLoS ONE, 2016, 11(3): e0151944 (doi: 10.1371/journal.pone.0151944).

12. A m a n n R.I., Ludwig W., S c hle i f e r K.H. Phylogenetic identification and in situ detection of individual microbial cells without cultivation. Microbiol. Rev., 1995, 59: 143-169.

13. Instruktsiya po sanitarno-mikrobiologicheskomu kontrolyu tushek, myasa ptitsy, ptitseproduktov, yaits $i$ yaitseproduktov na ptitsevodcheskikh i pererabatyvayushchikh predpriyatiyakh [Instruction for the sanitary-microbiological control of poultry carcasses, meat, eggs and egg products in the poultry production and processing enterprises]. Moscow, 1990 (in Russ.).

14. Maniat is T., Frits ch E. F., S a mb rook J. Molecular cloning: a laboratory manual. Cold Spring Harbor, NY, 1982.

15. van der Wielen P.W.J.J., Keuzenkamp D.A., Lipman L.J.A., van Kna pe n F., B i e st e rve ld S. Spatial and temporal variation of the intestinal bacterial community in commercially raised broiler chickens during growth. Microbiol. Ecol., 2002, 44: 286-293.

16. Maiorka A., Dahlke F., de Azevedo Morgulis M.S.F. Broiler adaptation to post-hatching period. Ciencia Rural, 2006, 36: 701-708.

17. Kize rwette r-Swida M., B inek M. Bacterial microflora of the chicken embryos and newly hatched chicken. J. Animal Feed Sci., 2008, 17: 224-232 (doi: 10.22358/jafs/66602/2008).

18. $\mathrm{B} \mathrm{a} \mathrm{b} \mathrm{a} \mathrm{c} \mathrm{a} \mathrm{Z.} \mathrm{Isolation} \mathrm{of} \mathrm{bacterial} \mathrm{pathogens} \mathrm{from} \mathrm{dead} \mathrm{in-shell} \mathrm{chicken} \mathrm{embryos} \mathrm{from} \mathrm{local}$ hatcheries. J. Vet. Sci. Technol., 2014, 5: 170-171.

19. Rossi D.A., Fonseca B.B., de Melo R.T., da Silva Felipe G., da Silva P.L., M e nd o n ça E.P., F i l gu e i r a s A.L., B e l e t t i M.E. Transmission of Campylobacter coli in chicken embryos. Brazil. J. Microbiol., 2012, 43(2): 535-543 (doi: 10.1590/S151783822012000200014).

20. Nakphaichit M., Thanomwongwattana S., Phraephaisarn C., Sa ka moto N., Ke aw sompong S., Nakayama J., Nitisinprasert S. The effect of including Lactobacillus reuteri KUB AC5 during post hatch feeding on the growth and ileum microbiota of broiler chickens. Poultry Sci., 2011, 12(90): 2753-2765 (doi: 10.3382/ps.2011-01637).

21. L o w d e r B.V., Fit z g e r a ld J.R. Human origin for avian pathogenic Staphylococcus aureus. Virulence, 2010, 4(1): 283-284 (doi: 10.4161/viru.1.4.11838).

22. B ibe rste i n E.L., Hi rs h D.C. Bordetella in veterinary microbiology. Reino Unido, Blackwell Sci., Oxford, 1999: 148-150.

23. Gong J., Forster R.J., Yu H., Chambers J.R., Sabour P.M., Whe atcroft R., 
$\mathrm{Ch}$ e n S. Diversity and phylogenetic analysis of bacteria in the mucosa of chicken ceca and comparison with bacteria in the cecal lumen. FEMS Microbiol. Lett., 2002, 208(1): 1-7 (doi: 10.1111/j.1574-6968.2002.tb11051.x).

24. Torok V.A., H ugh es R.J., M ik k e ls e n L.L. Identification and characterization of potential performance-related gut microbiota in broiler chickens across various feeding trials. Appl. Environ. Microbiol., 2011, 77(17): 5868-5878 (doi: 10.1128/AEM.00165-11).

25. Rinttila T., Apajalahti J. Intestinal microbiota and metabolites - implications for broiler chicken health and performance. J. Appl. Poultry Res., 2013, 22(3): 647-658 (doi: 10.3382/japr.2013-00742). 